\title{
A near-infrared survey of the entire R Coronae Australis cloud ${ }^{\star}$
}

\author{
M. Haas, F. Heymann, I. Domke, H. Drass, R. Chini, and V. Hoffmeister
}

Astronomisches Institut, Ruhr-Universität Bochum, Universitätsstraße 150, 44801 Bochum, Germany

Received 11 April 2008 / Accepted 25 June 2008

\section{ABSTRACT}

\begin{abstract}
Aims. To understand low- to intermediate-mass star-formation in the nearby R Cr A molecular cloud, we try to identify the stellar content that is accessible with near-infrared observations.

Methods. We obtained a $J H K_{\mathrm{s}}$ band mosaic of $\sim 10^{\prime} \times 60^{\prime}$ covering the entire R CrA molecular cloud with unprecedented sensitivity. We present a catalogue of about 3500 near-infrared sources fainter than the saturation limit $K_{\mathrm{s}} \sim 10$ mag, reaching $K_{\mathrm{s}} \sim 18$ mag. We analysed the extended sources by inspecting their morphology and point sources by means of colour-colour and colour-magnitude diagrams. Additionally, we compared the extinction inferred from the NIR data with the line-of-sight dust emission at $1.2 \mathrm{~mm}$. Sources towards high dust emission but relatively low $H-K_{\mathrm{s}}$ show a projected mm-excess; these sources are either immediately surrounded by cold circumstellar material or, if too red to be a true foreground object, they are embedded in the front layer of the $1.2 \mathrm{~mm}$ emitting dust cloud. In both cases they are most likely associated with the cloud.

Results. By means of the projected mm-excess technique we find 33 new faint near-infrared sources deeply embedded in the Coronet cluster around R CrA, for which so far about 20 bright infrared stars have been known. In contrast to the Coronet region, both the northwestern dust ridge and the southeastern cloud condensation "C" appear to be devoid of associated stars detectable with our near-infrared data. Furthermore, about a dozen sources, which are spread over the entire molecular cloud region, exhibit a possible $K$-band excess, but only with marginal statistical significance $(<3 \sigma)$, so that we do not consider the indicated $K$-band excess as real. Finally, while the Herbig-Haro-like objects seen on our maps are concentrated around the Coronet, we find four new nebulae also located farther down to the southeast. At the position of IRAS 18595-3712, an X-shaped bipolar nebula is resolved; its exciting star is hidden behind an edge-on disc.

Conclusions. The deep near-infrared survey of the entire R CrA molecular cloud strengthens the evidence for the Coronet being the region where most of the young stars are found. Our results are consistent with earlier predictions that the R CrA cloud has fragmented into sub-condensations at different star-forming stages.
\end{abstract}

Key words. stars: formation - stars: pre-main sequence - stars: low-mass, brown dwarfs - infrared: stars

\section{Introduction}

At a distance of about $170 \mathrm{pc}$ (Knude \& Hog 1998), the R Cr A molecular cloud (Rossano's cloud A, 1978) is among the closest star-forming regions. Surveys of this cloud have revealed a population of young intermediate- to low-mass stars, through the presence of $\mathrm{H} \alpha$ emission (e.g. Marraco \& Rydgren 1981; Fernández \& Comerón 2001) or as X-ray sources (e.g. Forbrich \& Preibisch 2007). Near-infrared (NIR) observations detected the Coronet, a small cluster of embedded sources around R Cr A itself (Taylor \& Storey 1984). Further NIR observations by Wilking et al. (1997) focussed on the Coronet region and analysed sources down to $K=15 \mathrm{mag}$, which - due to the large extinction in most parts of the observed region - yields a mass detection limit of about $1 M_{\odot}$. More deeply embedded sources have been identified by radio continuum (Brown 1987), midinfrared (Olofsson et al. 1999), and millimetre (Chini et al. 2003; Nutter et al. 2005) observations. The young $\left(10^{3}-10^{5} \mathrm{yrs}\right)$ stars drive various outflows that are observed either as Herbig-Haro objects (e.g. Hartigan \& Graham 1987; Wang et al. 2004) or as molecular outflows (e.g. Anderson et al. 1997). Using the Swedish ESO Submillimetre Telescope (SEST), the gas and dust content of the entire R Cr A cloud having an extent of $10^{\prime} \times 50^{\prime}$

* Table A1 is only available in electronic form at the CDS via anonymous ftp to cdsarc.u-strasbg.fr (130.79.128.5) or via http://cdsweb.u-strasbg.fr/cgi-bin/qcat?]/A+A/488/987 has been mapped in $\mathrm{C}^{18} \mathrm{O} J=1-0$ by Harju et al. (1993) and at $1.2 \mathrm{~mm}$ with SIMBA (Chini et al. 2003). At galactic coordinates $l=0^{\circ}, b=-18^{\circ}$ any fore- and background contamination should be negligible.

The R Cr A molecular cloud lies at the apex of a several degrees large cometary-shaped flocculent reflection nebula. As discussed by Harju et al. (1993), the cloud may have been influenced about 10-13 Myr ago by the expanding H I shell of the Upper Centaurus-Lupus OB association located west-northwest of $\mathrm{R} \mathrm{CrA}$. Then a rotating disc-like cloud seen roughly edgeon could have fragmented into several sub-condensations that further contract to form stars. The central condensation A, the Coronet, houses the currently observed young sources, while the neighbouring regions northwest (NW), and southeast (C) show virtually no ongoing star formation. The dense region $\mathrm{C}$ was predicted to be a site for future star formation (Harju et al. 1993), and in fact a cold cloud core has been detected there (Chini et al. 2003). On the other hand, it is puzzling why the dust ridge northwest of the Coronet lacks signs of recent star formation. This stimulated our aim of identifying the stellar content along the entire cloud down to at least $0.1 M_{\odot}$ (spectral type M7V - M8V, see Landolt-Börnstein 1982).

Because of the expected high extinction at optical wavelengths, we performed a deep and spatially complete NIR survey of the R Cr A cloud. We gave special emphasis on covering the outermost regions NW and C, which in the NIR had only been 
surveyed by $2 \mathrm{MASS}$, and to map the Coronet with at least 2 mag higher sensitivity than was achieved by Wilking et al. (1997). Here we present the data and perform a first analysis with respect to the $1.2 \mathrm{~mm}$ dust continuum map. Further comparison of the NIR data with MIR data will be provided in a follow-up publication.

\section{Observations and data reduction}

Using ISPI at the CTIO 4-m Blanco telescope with 10' FOV at a pixel size of $0 . ' 3$, we obtained $J, H, K_{\mathrm{s}}$ maps of six fields labeled NW, A, A3, B, D, C covering the entire R Cr A molecular cloud (Fig. 1).

The $J$ and $H$-band observations of field A, requiring long integrations under good conditions, were lost due to poor weather, even during a second observing campaign in 2005. While limited NIR colour information could be derived for the Coronet from the overlap with the neighbouring fields A3 and NW and from the $J$ and $H$-band data by Wilking et al. (1997), the deep $K_{\mathrm{s}}$-band image of field A allowed us to perform morphological studies and will be useful for future comparisons, e.g. with the Spitzer 3.6-24 $\mu \mathrm{m}$ maps that cover our fields A, A3, and B, but only $50 \%$ of fields D and NW (PI Giovanni Fazio, Lori Allen et al. in preparation). Essentially each source seen on the Spitzer 3.6 and $4.5 \mu \mathrm{m}$ map was also detected in our $K_{\mathrm{s}}$-band image of field A.

The ISPI integration times per field were adjusted to the extinction estimated from the $1.2 \mathrm{~mm}$ SIMBA map (Chini et al. 2003). The observational parameters and the resulting sensitivities are listed for each field in Table 1 . The field size refers to the area covered by all three filters. Because the observations at different filters had small offsets, the resulting common field sizes differ slightly from field to field. The detection limit, as well as the limiting sensitivity of sources with $\mathrm{rms}<0.2 \mathrm{mag}$, refer to the mean of the faintest three objects in the field-filter combination. We determined the $100 \%$ completeness limit for each field and filter from the magnitude histograms. The completeness limits are uncertain, because the maps are not homogeneous; for example near R CrA large areas are saturated or affected by spikes.

Each mosaic has been observed with several dither cycles $\left(20^{\prime \prime}\right)$ under moderately photometric but good seeing conditions $\left(1^{\prime \prime}-1{ }^{\prime \prime} 5\right)$. We did not collect extra sky frames, but instead constructed the mean sky frames from the field images themselves. Therefore, our photometry is limited to objects with an extent $<20^{\prime \prime}$. Dome flats were taken at the beginning and end of each night.

Standard data reduction was performed using the software packages for the ISPI instrument implemented in the Image Reduction and Analysis Facility (IRAF). This includes astrometric corrections across each field. The sources were extracted using the DAOFIND and DAOPHOT tools and for comparison using the SEXTRACTOR tool (Bertin \& Arnouts 1996). The astrometric and photometric calibration was performed using 2MASS stars in the fields. The astrometric accuracy was conservatively estimated to be better than $1^{\prime \prime}$, while the photometric uncertainty depends on the brightness of the sources; we applied a cut of rms $=0.4 \mathrm{mag}$ for the final catalogues. For sources located in the overlap of two adjacent fields we list the mean photometry from both fields.

We inspected the individual frames for sources with extended morphology. They are listed in Table 2, with photometry derived from suitably chosen apertures. Point sources are listed with PSF photometry in Table A1, available only in electronic form, and having the same layout as Table2. Sources brighter
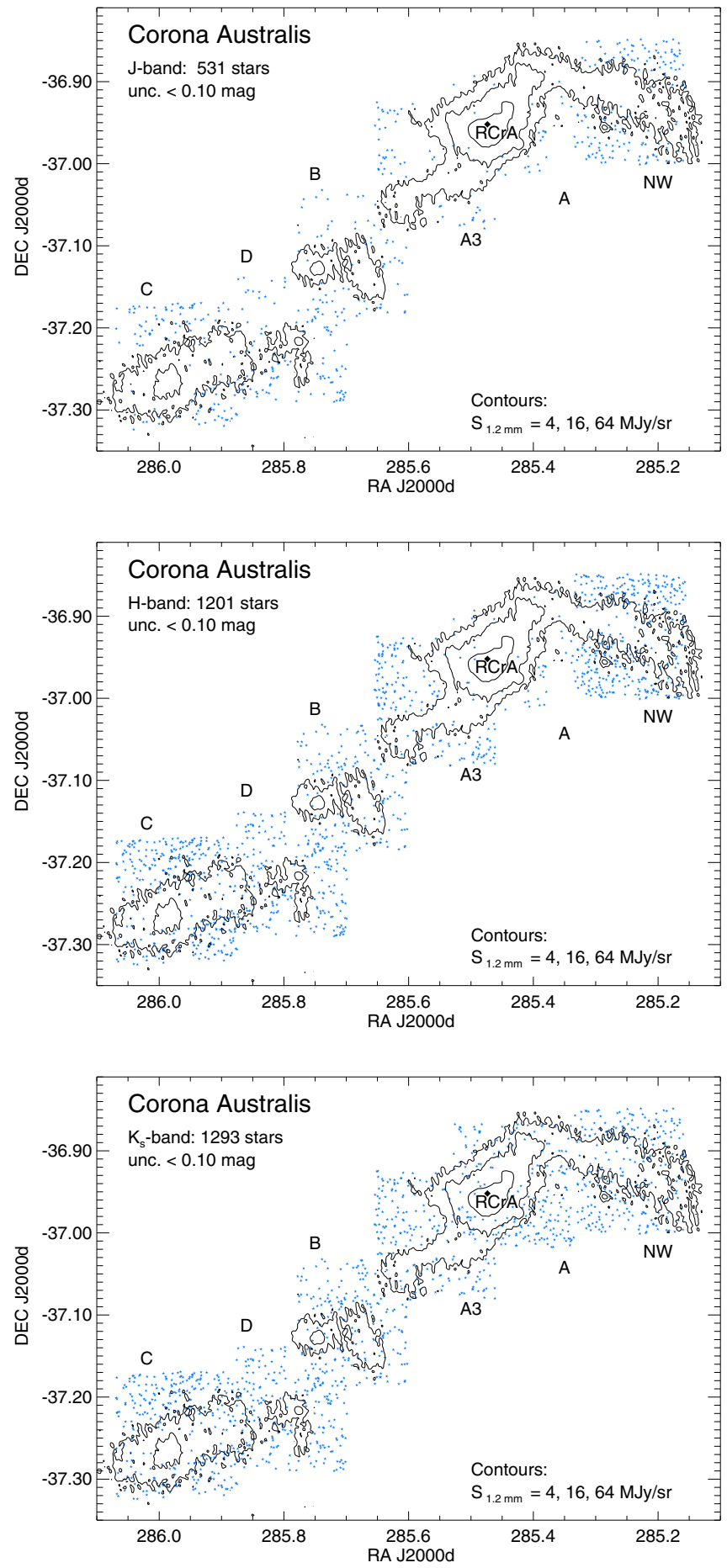

Fig. 1. Distribution of IR sources with uncertainty rms $<0.1$ mag towards the $\mathrm{R} \mathrm{Cr}$ A cloud. The $1.2 \mathrm{~mm}$ contours from the SIMBA map are superimposed. The fields are labelled (NW, A, A3, B, D, C). R Cr A in the overlap of fields $\mathrm{A}$ and $\mathrm{A} 3$ is marked with a diamond.

than $K_{\mathrm{s}} \sim 10$ mag are saturated (outside the linear range of the detector) and therefore excluded. Tables 2 and A1 also list the SIMBA surface brightness $S_{1.2 \mathrm{~mm}}$ averaged over $28^{\prime \prime} \times 28^{\prime \prime}$ at the sky position of the sources (SIMBA HPBW $=24^{\prime \prime}$ ). The SIMBA surface brightness does not necessarily refer to isolated $1.2 \mathrm{~mm}$ point sources, rather it is derived from the mostly broad and smooth $1.2 \mathrm{~mm}$ emission towards each NIR source, in order to provide a measure of the dust column density in the direction 
Table 1. Observational parameters (top) and sensitivities (bottom).

\begin{tabular}{|c|c|c|c|c|c|}
\hline Field & \multicolumn{2}{|c|}{ RA J2000d } & Dec J2000d & Field size & Obs. date \\
\hline $\mathrm{C}$ & \multicolumn{2}{|c|}{285.971985} & -37.246700 & $9^{\prime} .5 \times 99^{\prime} .3$ & 31 July 2004 \\
\hline D & \multicolumn{2}{|c|}{285.788513} & -37.214447 & $8: 6 \times 9 ! .1$ & 29 July 2004 \\
\hline B & \multicolumn{2}{|c|}{285.689514} & -37.108849 & $8^{\prime} .5 \times 9^{\prime} .2$ & 29 July 2004 \\
\hline A3 & \multicolumn{2}{|c|}{285.559021} & -37.003448 & $9^{\prime} .4 \times 9^{\prime} .5$ & 29 July 2004 \\
\hline A & \multicolumn{2}{|c|}{285.429504} & -36.941799 & $9^{\prime} .4 \times 9^{\prime} .1$ & 30 July 2004 \\
\hline NW & \multicolumn{2}{|c|}{285.247498} & -36.925301 & $8.9 \times 9.2$ & 29 July 2004 \\
\hline \multicolumn{2}{|c|}{ Field Filter } & $\begin{array}{l}\text { Integr. } \\
\text { time [s] }\end{array}$ & $\begin{array}{c}\text { Detect. } \\
\text { limit [mag] }\end{array}$ & $\begin{array}{c}\mathrm{rms}<0.2 \\
\text { limit }[\mathrm{mag}]\end{array}$ & $\begin{array}{l}\text { Complete. } \\
\text { limit [mag] }\end{array}$ \\
\hline $\mathrm{C}$ & $J$ & 2000 & 20.3 & 19.0 & 17.6 \\
\hline $\mathrm{C}$ & $H$ & 270 & 19.1 & 18.3 & 17.1 \\
\hline $\mathrm{C}$ & $K_{\mathrm{s}}$ & 120 & 18.4 & 17.6 & 16.8 \\
\hline D & $J$ & 375 & 19.1 & 18.4 & 17.1 \\
\hline D & $H$ & 270 & 18.8 & 18.1 & 16.8 \\
\hline D & $K_{\mathrm{s}}$ & 120 & 17.7 & 17.1 & 16.3 \\
\hline B & $J$ & 375 & 18.8 & 18.3 & 16.9 \\
\hline B & $H$ & 270 & 18.2 & 17.8 & 17.0 \\
\hline B & $K_{\mathrm{s}}$ & 120 & 17.5 & 17.2 & 16.4 \\
\hline A3 & $J$ & 375 & 19.3 & 18.7 & 18.0 \\
\hline A3 & $H$ & 270 & 19.0 & 18.5 & 17.1 \\
\hline A3 & $K_{\mathrm{s}}$ & 120 & 17.6 & 17.3 & 16.2 \\
\hline A & $K_{\mathrm{s}}$ & 6800 & 18.2 & 17.7 & 16.9 \\
\hline NW & $J$ & 1000 & 19.4 & 18.5 & 17.3 \\
\hline NW & $H$ & 600 & 19.0 & 18.3 & 17.0 \\
\hline NW & $K_{\mathrm{s}}$ & 120 & 17.2 & 16.8 & 16.0 \\
\hline
\end{tabular}

of the star. The dust producing this emission may be located in front, nearby, or behind the star.

\section{Results and discussion}

Figure 1 shows for each filter $J, H$, and $K_{\mathrm{s}}$ the sky position of the point sources having photometric rms $<0.1 \mathrm{mag}$, as well as the SIMBA $1.2 \mathrm{~mm}$ contours superimposed. The most striking result from Fig. 1 is the steep decline in source density towards sky regions with bright $1.2 \mathrm{~mm}$ emission, i.e. highest dust column density. This is illustrated in Fig. 2. The decline in stellar surface density is most prominent in the $J$-band and steepens considerably in the $H$-band above $S_{1.2}>20 \mathrm{MJy} / \mathrm{sr}$; the $K_{\mathrm{s}}$-band appears less affected. However, even at $2.2 \mu \mathrm{m}$ the dusty central regions of the $\mathrm{R} \mathrm{Cr}$ A cloud, in particular towards fields $\mathrm{A}$ and $\mathrm{C}$, turn out to be opaque for background stars, dimming them below $K \sim 18$ mag. Stars seen towards these opaque regions may be foreground stars or located within the front layers of the cloud. On the other hand, most of the sources towards low $1.2 \mathrm{~mm}$ surface brightness are likely background stars.

To identify stars associated with the R Cr A cloud, we applied three strategies:

- projected millimetre excess sources (Sect. 3.1);

- $K_{\mathrm{s}}$-band excess sources (Sect. 3.2);

- nebulae and extended objects (Sect. 3.3).

\subsection{Projected millimetre excess sources}

To determine the cloud membership for those point sources with $H$ and $K_{\mathrm{s}}$-band photometry available, and independent of $J$-band detection, we apply a new strategy here involving the $1.2 \mathrm{~mm}$ data. Basically we follow the well-known fact that, for a background source, the extinction derived from the dust column, i.e. the $1.2 \mathrm{~mm}$ brightness, should match the $H-K_{\mathrm{s}}$ colour of that

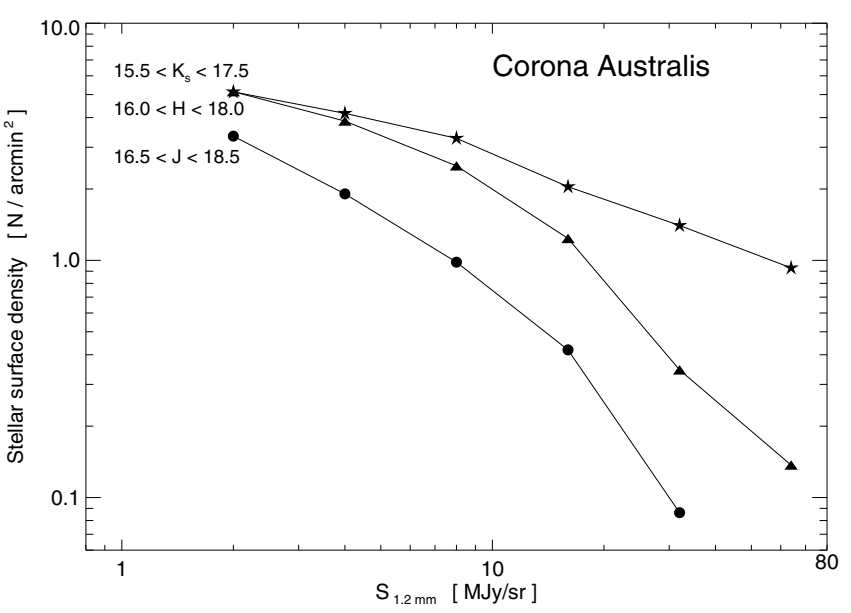

Fig. 2. Surface density of sources with rms $<0.2$ mag as a function of wavelength and the SIMBA $1.2 \mathrm{~mm}$ emission towards these sources. For $J$ and $H$, only five fields are considered, while $K_{\mathrm{s}}$ refers to all six fields including field A.

source. The observed reddening (extinction) is correlated with the hydrogen column density, and - via the dust/gas ratio - also with the dust column density $\left(A_{\mathrm{V}} / N_{\mathrm{H}} \sim 5.6 \times 10^{-22} \mathrm{mag} / \mathrm{cm}^{-2}\right.$ - Seward 1999). We trawl the cloud members using the cloud's dust emission as a fishing net: if a source lies towards a high dust column but has too a small "deficient" extinction as derived from $H-K_{\mathrm{s}}$, then this source cannot be located behind the dust cloud, but instead it must be a foreground source or a cloud member. Such a "projected mm-excess" source is either surrounded by cold circumstellar material typical for a very young object likely associated with the parent molecular cloud, or it must be embedded in the front layer of the dust cloud, if the $H-K_{\mathrm{s}}$ derived extinction is too high for a true foreground source. The projected mm-excess technique is particularly suited to identifying cloud sources without $K$-band excess.

Figure 3 shows the distribution of the $1.2 \mathrm{~mm}$ surface brightness versus $H-K_{\mathrm{s}}$. Here we plot all sources with photometric uncertainty $<0.2 \mathrm{mag}$. The bulk of the sources lie at both low $S_{1.2}<5 \mathrm{MJy} / \mathrm{sr}$ and $H-K_{\mathrm{s}}<1$, consistent with moderately reddened background stars off the cloud cores. There are also many objects exhibiting larger $H-K_{\mathrm{s}}$ and considerable $S_{1.2}>8 \mathrm{MJy} / \mathrm{sr}$. These objects are seen in projection towards the dense dust cloud. The red squares show the median of $S_{1.2}$ along $H-K_{\mathrm{s}}$ bins (with upper and lower quartiles as error bars). The thick solid line represents the extinction $A_{\mathrm{V}}$ predicted from the dust column, using standard dust properties ( $\kappa=0.37 \mathrm{~cm}^{2} \mathrm{~g}^{-1}$, Krügel 2003). The results derived below are quite independent of the detailed dust properties. The $A_{\mathrm{V}}$ line is not a fit to the median data points, but agrees well with them up to $H-K_{\mathrm{s}}<1.5$. At higher $H-K_{\mathrm{s}}$, the agreement of the data with the $A_{\mathrm{V}}$ line improves after omitting the stars with extremely high $S_{1.2}>25 \mathrm{MJy} / \mathrm{sr}$.

In the following we distinguish between sources with extremely bright $S_{1.2}>25 \mathrm{MJy} / \mathrm{sr}$, which we call probable members, and sources with intermediate $S_{1.2}>8 \mathrm{MJy} / \mathrm{sr}$, which we call possible members.

\subsubsection{Probable members}

There are only two spots outside of the Coronet that have $S_{1.2}>$ $25 \mathrm{MJy} / \mathrm{sr}$ : VV CrA (MMS 24) and MMS 23 (see Sect. 3.3) 
Table 2. Extended objects. Positions and photometry from suitably chosen apertures, errors are given in brackets.

\begin{tabular}{|c|c|c|c|c|c|c|}
\hline RA J2000d & Dec J2000d & $J[\mathrm{mag}]$ & $H[\mathrm{mag}]$ & $K_{\mathrm{s}}[\mathrm{mag}]$ & $\mathrm{S} 1.2 \mathrm{~mm}[\mathrm{MJy} / \mathrm{sr}]$ & Remarks, size ["] \\
\hline 285.508212 & -36.899951 & & & $15.80(0.18)$ & $-0.8(1.1)$ & Star with nebula ${ }^{a}, \sim 3^{\prime \prime}$ \\
\hline 285.512850 & -37.002804 & $\ldots$ & $16.32(0.09)$ & $14.34(0.15)$ & $11.8(2.2)$ & Star with nebula ${ }^{a}, \sim 3^{\prime \prime}$ \\
\hline 285.522940 & -36.910680 & $\ldots$ & & $11.60(0.30)$ & $-0.3(0.9)$ & HH $99^{a}, \sim 25^{\prime \prime}$ \\
\hline 285.552670 & -36.945956 & $16.26(0.09)$ & $15.20(0.08)$ & $13.78(0.08)$ & $7.7(1.8)$ & $\mathrm{HH} 735, \sim 10^{\prime \prime}$ \\
\hline 285.612310 & -37.175960 & $16.64(0.06)$ & $15.09(0.05)$ & $14.38(0.05)$ & $0.1(0.7)$ & Star with nebula, $\sim 5^{\prime \prime}$ \\
\hline 285.702890 & -37.238030 & $16.82(0.09)$ & $15.24(0.09)$ & $14.74(0.04)$ & $0.9(1.7)$ & Star with nebula, $\sim 4^{\prime \prime}$ \\
\hline 285.744250 & -37.126140 & $\ldots$ & $14.32(0.10)$ & $12.60(0.07)$ & $32.8(7.3)$ & Isabelle Nebula, MMS 23, 20" \\
\hline 285.883453 & -37.286964 & $15.32(0.15)$ & $14.12(0.15)$ & $13.91(0.15)$ & $0.4(0.6)$ & Star with nebula, $\sim 5^{\prime \prime}$ \\
\hline
\end{tabular}

${ }^{a}$ Also seen by Wilking et al. (1997).

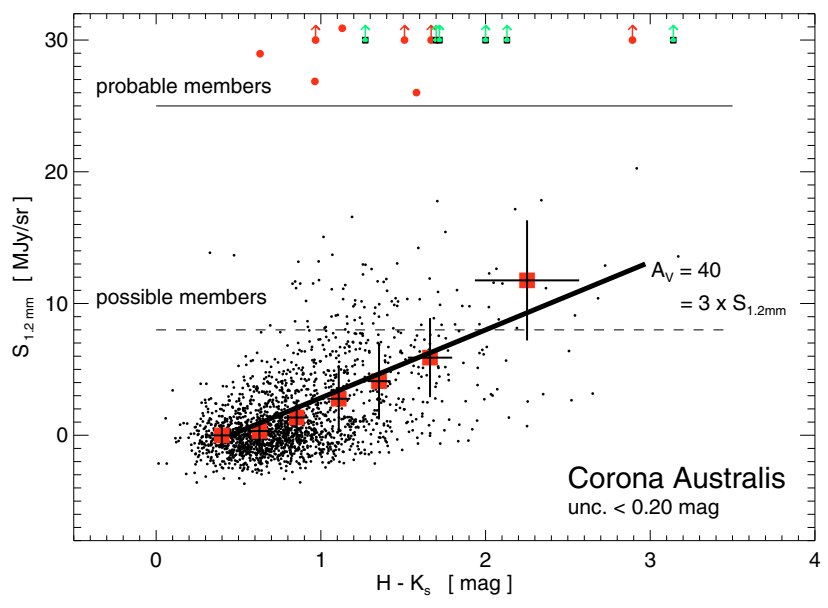

Fig. 3. $1.2 \mathrm{~mm}$ surface brightness versus $H-K_{\mathrm{s}}$. Sources above the solid line $S_{1.2}=25 \mathrm{MJy} / \mathrm{sr}$ are considered to be probable members, and sources above the long-dashed line $S_{1.2}=8 \mathrm{MJy} / \mathrm{sr}$ are called possible members. The thick solid line indicates $A_{V}$ adopting standard dust properties; it is not a fit to the median data points (red squares).

located in fields $\mathrm{D}$ and $\mathrm{B}$, respectively. The dust emission in these bright spots $\left(S_{1.2} \sim 30 \mathrm{MJy} / \mathrm{sr}\right)$ is probably directly attributed to the PMS stars (Wilking et al. 1992; Chini et al. 2003; Nutter et al. 2005). The source IRS 2, coincident with MMS 9, is saturated ( $K=7.16 \mathrm{mag}$ ). These three sources are not considered further here.

There are 14 sources with $S_{1.2}>25 \mathrm{MJy} / \mathrm{sr}$ far above the $A_{\mathrm{V}}$ line in Fig. 3. All of them are located towards the Coronet (Fig. 4). In our $K_{\mathrm{s}}$ image, the immediate neighbourhood of $\mathrm{R} \mathrm{CrA}$ is crowded with bright nebulous features that make any detection of faint sources difficult. Therefore, our new detected $K_{\mathrm{s}}$ sources only appear at some distance from $\mathrm{R} \mathrm{CrA}$. The 14 probable members are not distinct $1.2 \mathrm{~mm}$ point sources in the SIMBA map, but their $1.2 \mathrm{~mm}$ flux is due to extended cloud emission towards their line of sight. If they were background stars behind the bright $1.2 \mathrm{~mm}$ dust emission, their light would suffer an extinction of $75<A_{\mathrm{V}}<500$. In contrast, the extinction derived from $H-K_{\mathrm{s}}$ is much lower $\left(2<A_{\mathrm{V}}<40\right)$, but too high for a true foreground source. Therefore, they must be embedded in the front layer of the cloud.

We have also considered the location of known members of the R CrA association in Fig. 3. Their membership has been inferred, for instance, from $\mathrm{H} \alpha$ (Marraco \& Rydgren 1981; Fernandéz \& Comerón 2001) or X-ray emission (Forbrich \& Preibisch 2007). These sources are not exclusively located towards regions with high $1.2 \mathrm{~mm}$ dust emission, but many of them lie in regions around the cloud having a low projected dust

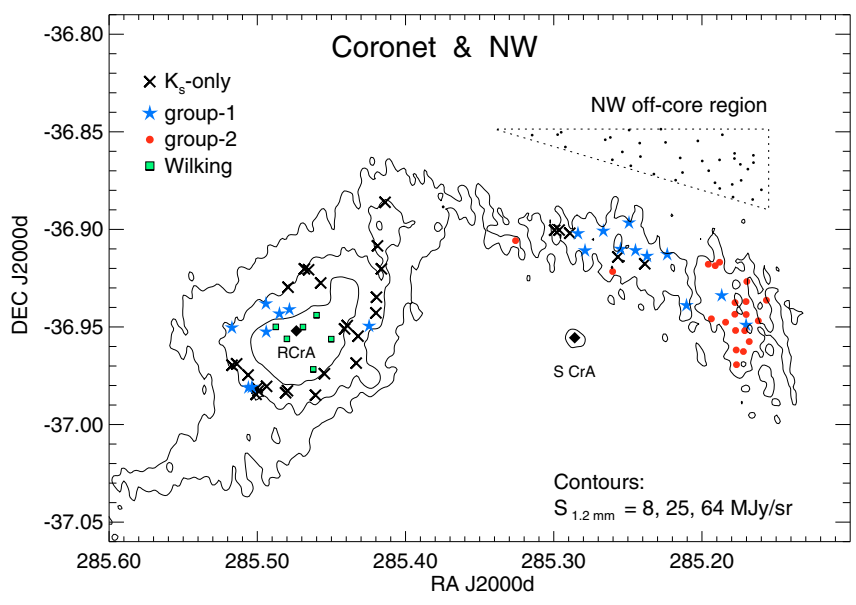

Fig. 4. Sky position of the probable members and the possible NW cloud members. All probable members are located towards the Coronet around R CrA (filled diamond). Some sources lie near saturated areas or spikes in our $K_{\mathrm{s}}$-image, and their photometry was taken from Wilking et al. (1997). The possible members are located towards the core of the NW dust ridge, north and west of S CrA (filled diamond). The subdivision of stars into group- $1 /-2$ has been derived from the colour-magnitude diagram (Fig. 7). The dotted triangle encircles the stars in the NW off-core region used for comparisons.

column. Also, the known members exhibit a wide range of extinction properties (range in $H-K_{\mathrm{s}}$ ). From this it is clear that the known members are more or less randomly distributed thoughout the entire range depicted in Fig. 3. We did not overplot them in this figure with extra symbols, to avoid confusion with the other symbols. While known members are located both towards and around the cloud, the projected mm-excess technique is limited to identifying only the sources located in projection against the cloud.

Figure 5 shows the $K_{\mathrm{s}}$ vs. $H-K_{\mathrm{s}}$ colour-magnitude diagram of the probable members. For comparison, the unreddened main sequence at $170 \mathrm{pc}$ distance and the extinction vector is plotted ${ }^{1}$. The probable members are stars of intermediate to low mass down to about spectral type M0 $\left(0.5 M_{\odot}\right.$, see Landolt-Börnstein 1982). If they had a strong K-band excess, some sources could even have lower mass, but we did not find evidence of a strong $K$-excess as shown in Sect.3.2. Comparing our data with the

1 The main sequence was taken from Schmidt-Kaler (1982) for spectral type A0 to M4 and from Dahn et al. (2002) for M0 to L0. The two references exhibit a discrepancy of about $1 \mathrm{mag}$ for M0 stars. To bridge this gap and to provide a smooth presentation in the plot, we stretched the Schmidt-Kaler main sequence evenly between G0 and M4, fitting the more actual M0 magnitudes of Dahn et al. In colour-colour plots, the location of the main sequence is virtually not affected. 


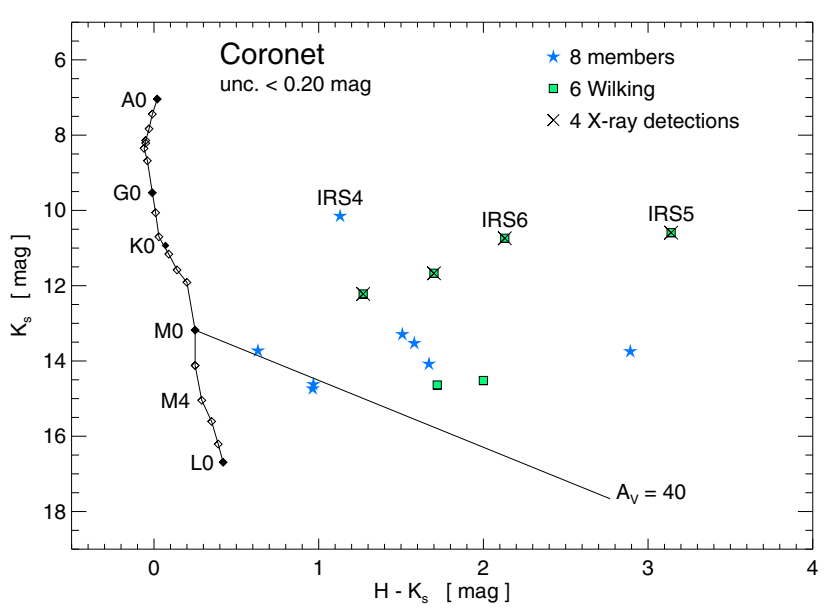

Fig. 5. Colour-magnitude diagram of the probable members, i.e. projected mm-excess sources with $S_{1.2}>25 \mathrm{MJy} / \mathrm{sr}$.

Chandra results by Forbrich \& Preibisch (2007), only the brightest stars $\left(K_{\mathrm{s}}<12 \mathrm{mag}\right.$, all being young) are detected in the X-ray map. This suggests that deeper X-ray observations are required to draw further conclusions about the accretion activity of our faint probable members. The 9 sources fainter than $K_{\mathrm{s}}=13 \mathrm{mag}$ were not known to be associated with the cloud. They are not detected by 2 MASS. The mm-excess strategy enables us to establish their cloud membership. The probable members are listed in Table 3.

Next we consider the numerous faint $K_{\mathrm{s}}$-only sources detected in field A without appropriate $H$-band photometry available. Figure 6 shows the $K_{\mathrm{s}}$ brightness vs. $S_{1.2}$. For comparison the extinction vector and sources with $H$ detection in the other fields (C, D, B, A3, NW) are plotted. There are $24 K_{\mathrm{s}}$-only sources with projected mm-excess $S_{1.2}>25 \mathrm{MJy} / \mathrm{sr}$. Because they have $K_{\mathrm{s}} \sim 15-18 \mathrm{mag}$, our sources are not seen in deep optical images $(R \sim 22.5 \mathrm{mag}, I \sim 20.5 \mathrm{mag}$, López Martí et al. 2005). The colours $R-K_{\mathrm{s}}>4.5$ and $I-K_{\mathrm{s}}>2.5$ rule out the possibility of unreddened foreground stars. If they were located behind the very bright $1.2 \mathrm{~mm}$ dust emission $\left(S_{1.2}>25 \mathrm{MJy} / \mathrm{sr}\right.$ corresponding to $A_{\mathrm{V}}>75$ ), they would form an ensemble of background stars earlier than spectral type A0 with a projected concentration towards the Coronet. Because this is unlikely and not consistent with our analysis of various off-cloud regions, we conclude that the 24 newly-found infrared sources with projected mm-excess are deeply embedded in the Coronet.

Compared to probable members with $H$ photometry available, the $K_{\mathrm{s}}$-only sources are fainter (Fig. 6, Table 3 ) and probably more reddened. For an adopted extinction $A_{\mathrm{V}} \sim 30-40^{\mathrm{m}}$ (see Fig. 5), their dereddened brightness would be about $K_{\mathrm{s}}=12-15 \mathrm{mag}$. If not Herbig-Haro knots, then as (presumably PMS) K-stars and M-dwarfs, they would represent the lower mass extension of the known Coronet members. Because theoretical concepts and numerical simulations find that lower mass stars evolve more slowly than higher mass stars, one may expect that the low-mass end of the $K_{\mathrm{s}}$-only sources will show a strong disc excess at mid-IR wavelengths.

\subsubsection{Possible members}

In contrast to the Coronet region, both the northwestern dust ridge and the southeastern cloud condensation $\mathrm{C}$ are much fainter at $1.2 \mathrm{~mm}$, so that we cannot simply trawl the associated

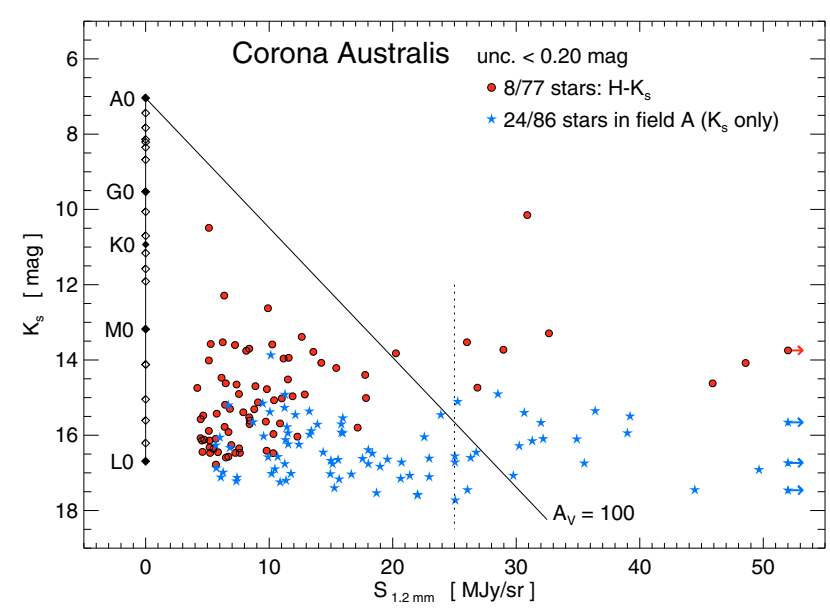

Fig. 6. $K_{\mathrm{s}}$ brightness versus $S_{1.2}$ : plotted are sources of field A having only $K_{\mathrm{s}}$ photometry. They are compared with sources detected in both $H$ and $K_{\mathrm{s}}$ in any of our fields, which are located towards $S_{1.2}>25 \mathrm{MJy} / \mathrm{sr}$ or have red $H-K_{\mathrm{s}}>1.5$.

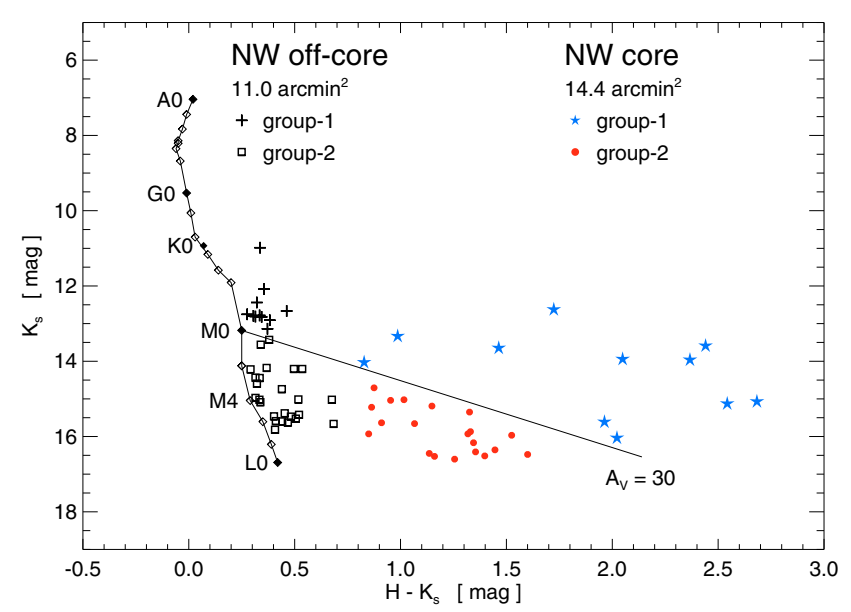

Fig. 7. Colour-magnitude diagram of the NW-core and NW-off-core. Group-1/-2 sources are defined to lie above/below the $A_{\mathrm{V}}$ line. The main sequence shifted to the distance of $\mathrm{R} \mathrm{CrA}$ is plotted only for illustrative purposes.

stars by means of a projected mm-excess. As a trial we investigate how far we can extend the technique to fainter dust surface brightness. We select possible members having $S_{1.2}>8 \mathrm{MJy} / \mathrm{sr}$ (Fig. 3). One possible member, the bright T Tauri star S CrA (MMS 1) having $S_{1.2} \sim 19 \mathrm{MJy} / \mathrm{sr}$, clearly is a cloud member. However, we have excluded this star from the following investigation, because its mm emission comes from a dust disc around the star and not from a smooth background (Wilking et al 1992; Chini et al. 2003; Nutter et al. 2005). In addition to the cores of $\mathrm{NW}$ and $\mathrm{C}$, we have analysed several off-core regions in fields $\mathrm{C}, \mathrm{D}, \mathrm{B}$ and $\mathrm{NW}$, in order to estimate the number and brightness of virtually unreddened background stars. All studied off-core regions yield similar number counts (Table 4) supporting that these regions are dominated by background stars. In the following we restrict the presentation of our analysis to the NW region, the results for region $\mathrm{C}$ are similar.

The possible members lie towards the core of the NW dustridge north and west of SCrA (Fig. 4). Figure 7 shows the colour-magnitude diagram of the possible members in the NW 
Table 3. Probable members found via the mm excess technique. Positions and photometry with errors given in brackets.

\begin{tabular}{|c|c|c|c|c|c|c|}
\hline RA J2000d & Dec J2000d & $J$ [mag] & $H$ [mag] & $K_{\mathrm{s}}[\mathrm{mag}]$ & $\mathrm{S} 1.2 \mathrm{~mm}[\mathrm{MJy} / \mathrm{sr}]$ & additional photometry reference \\
\hline 285.413788 & -36.886112 & $\ldots$ & $\ldots$ & $15.11(0.04)$ & $25.3(3.3)$ & \\
\hline 285.416229 & -36.920238 & $\ldots$ & $\ldots$ & $17.73(0.19)$ & $25.1(1.7)$ & \\
\hline 285.418884 & -36.908459 & $\ldots$ & $\ldots$ & $17.45(0.19)$ & $26.1(2.2)$ & \\
\hline 285.419556 & -36.934746 & $\ldots$ & $\ldots$ & $16.74(0.11)$ & $35.5(7.2)$ & \\
\hline 285.419922 & -36.942795 & $\ldots$ & $\ldots$ & $16.10(0.08)$ & $34.9(4.6)$ & \\
\hline 285.424561 & -36.949516 & $17.67(0.14)$ & $14.80(0.04)$ & $13.29(0.01)$ & $32.6(6.2)$ & \\
\hline 285.432007 & -36.954708 & $\ldots$ & $\ldots$ & $15.35(0.05)$ & $36.4(5.6)$ & \\
\hline 285.433258 & -36.968506 & $\ldots$ & $\ldots$ & $15.67(0.05)$ & $32.0(4.6)$ & \\
\hline 285.439301 & -36.949272 & $\ldots$ & $\ldots$ & $15.66(0.07)$ & $63.8(9.8)$ & \\
\hline 285.441071 & -36.951004 & $\ldots$ & $\ldots$ & $17.46(0.19)$ & $67.3(9.5)$ & \\
\hline 285.450104 & -36.956223 & $17.54(0.30)$ & $13.73(0.02)$ & $10.59(0.00)$ & $92.2(10.9)$ & Wilking $^{a}$, IRS5 Taylor \& Storey ${ }^{b}$ \\
\hline 285.454681 & -36.973965 & $\ldots$ & $\ldots$ & $16.74(0.11)$ & $60.6(15.1)$ & \\
\hline 285.457184 & -36.927448 & $\ldots$ & $\ldots$ & $16.92(0.13)$ & 49.7 ( 5.7) & \\
\hline 285.460022 & -36.943954 & $16.35(0.30)$ & $12.87(0.02)$ & $10.74(0.00)$ & $74.6(5.2)$ & Wilking $^{a}$, IRS6 Taylor \& Storey ${ }^{b}$ \\
\hline 285.460724 & -36.984917 & $\ldots$ & $\ldots$ & $15.40(0.07)$ & $30.7(5.8)$ & \\
\hline 285.462250 & -36.971722 & $\ldots$ & $16.52(0.18)$ & $14.52(0.12)$ & $103.2(16.1)$ & Wilking $^{a}$ \\
\hline 285.465393 & -36.920685 & $\ldots$ & $\ldots$ & $14.91(0.04)$ & $28.5(5.8)$ & \\
\hline 285.468170 & -36.920223 & $\ldots$ & $\ldots$ & $16.55(0.09)$ & $25.0(4.6)$ & \\
\hline 285.469177 & -36.950024 & $17.77(0.30)$ & $13.37(0.02)$ & $11.67(0.02)$ & $84.7(9.1)$ & Wilking $^{a}$, IRS9 Taylor \& Storey ${ }^{b}$ \\
\hline 285.478333 & -36.941036 & $\ldots$ & $15.59(0.14)$ & $14.62(0.03)$ & $45.9(6.9)$ & \\
\hline 285.479218 & -36.929649 & $\ldots$ & $\ldots$ & $16.72(0.11)$ & $25.0(4.4)$ & \\
\hline 285.479614 & -36.982685 & $\ldots$ & $\ldots$ & $17.45(0.19)$ & $44.5(10.0)$ & \\
\hline 285.480042 & -36.956081 & $\ldots$ & $13.49(0.22)$ & $12.22(0.14)$ & $170.7(23.6)$ & Wilking $^{a}$, IRS7 Taylor \& Storey ${ }^{b}$ \\
\hline 285.480865 & -36.983723 & $\ldots$ & & $15.50(0.05)$ & $39.2(8.6)$ & \\
\hline 285.485077 & -36.943222 & $\ldots$ & $15.75(0.18)$ & $14.08(0.03)$ & $48.6(9.0)$ & \\
\hline 285.487518 & -36.949936 & $\ldots$ & $16.36(0.28)$ & $14.64(0.08)$ & $87.9(19.2)$ & Wilking $^{a}$ \\
\hline 285.493683 & -36.980431 & $\ldots$ & $\ldots$ & $15.94(0.07)$ & $39.0(6.2)$ & \\
\hline 285.493988 & -36.952465 & $\ldots$ & $16.64(0.08)$ & $13.75(0.03)$ & $101.8(18.4)$ & \\
\hline 285.494202 & -36.938053 & $13.82(0.30)$ & $11.28(0.20)$ & $10.15(0.20)$ & 30.9 ( 5.4) & \\
\hline 285.499359 & -36.982796 & $\ldots$ & $\ldots$ & $17.07(0.15)$ & $29.8(5.0)$ & \\
\hline 285.500153 & -36.981281 & $\ldots$ & $\ldots$ & $16.09(0.08)$ & $32.2(5.1)$ & \\
\hline 285.500824 & -36.984539 & $\ldots$ & $\ldots$ & $16.46(0.09)$ & $26.8(5.1)$ & \\
\hline 285.503662 & -36.980827 & $16.06(0.04)$ & $14.36(0.02)$ & $13.73(0.03)$ & $29.0(5.9)$ & \\
\hline 285.506012 & -36.981091 & $17.74(0.18)$ & $15.70(0.06)$ & $14.74(0.04)$ & $26.9(5.3)$ & \\
\hline 285.506165 & -36.974621 & $\ldots$ & $\ldots$ & $16.15(0.08)$ & $31.3(5.1)$ & \\
\hline 285.513916 & -36.968899 & $\ldots$ & $\ldots$ & $16.28(0.09)$ & $30.2(4.2)$ & \\
\hline 285.517029 & -36.969604 & $\ldots$ & $\ldots$ & $16.60(0.12)$ & $26.3(3.6)$ & \\
\hline 285.517120 & -36.950371 & $\ldots$ & $15.11(0.04)$ & $13.53(0.01)$ & $26.0(2.2)$ & \\
\hline
\end{tabular}

${ }^{a}$ Wilking et al. (1997), ${ }^{b}$ Taylor \& Storey (1984).

core and the stars in the NW off-core region. All possible members have high enough extinction values $\left(A_{\mathrm{V}}>6 \mathrm{mag}\right)$ derived from $H-K_{\mathrm{s}}$ to rule out their being unreddened foreground stars. If the possible members were at the distance of R CrA, then they would be low-mass and even very low-mass stars (between spectral type $\mathrm{K} 0$ and $\mathrm{M} 0$, smaller than $\mathrm{M} 0$, respectively). However, we have to check that they are not background stars.

For the purpose of this check, we subdivide the NW core sources into group-1 and group-2 lying above/below the solid line in Fig. 7 and compare their extinction and sky position. Group-2 has, on average, smaller $H-K_{\text {s }}$, i.e. less extinction than group-1. Also, group-1 and group-2 sources populate mainly the right and left halves in Fig. 3. Thus at a given $1.2 \mathrm{~mm}$ flux as measured by the SIMBA beam, group- 2 sources suffer from less extinction. Group- 1 and $K_{\mathrm{s}}$-only sources concentrate towards the denser eastern region, but group-2 sources strongly populate the western border of the core at RA $<285.20$ (Fig. 4). This western border exhibits an abrupt change in the dust-to-gas properties: While the $1.2 \mathrm{~mm}$ emission roughly remains constant, the $\mathrm{C}^{18} \mathrm{O}$ emission declines rapidly at $\mathrm{RA}=285.20$. A natural explanation is that, at the cloud border, the dust distribution is clumpy with low filling factor not resolved in the SIMBA beam. Then a background object can easily be seen as group-2 source through a hole between dust clumps. This picture is consistent with the expectation that the wind from the Upper Centaurus-Lupus OB association corrodes the cloud's western front.

Furthermore, we compare the stellar surface density per magnitude bin for on- and off-core regions. After dereddening the stars in the colour-magnitude diagram, the stellar surface density $N$ per magnitude bin is statistically indistinguishable for on- and off-core (Table 4). Therefore, we suggest that our nearinfrared maps are able to permeate the NW core, so that most, if not all, of the sources detected towards the northwestern dust ridge are background stars.

In short, the results for region $\mathrm{C}$ are similar to those of $\mathrm{NW}$. Figure 8 shows the sky position of the possible members towards core $\mathrm{C}$ and the off-core field. Notably, in the immediate area around the extended $1.2 \mathrm{~mm}$ source MMS 25, no source is detected on our NIR maps. MMS 25 may be slightly too opaque for background stars to be detected on our maps, but class I-III stars in the cloud front layer should have been seen if they exist. Obviously, cloud core $\mathrm{C}$ contains at most very young proto-stars, which are too faint and embedded to be visible at near-infrared wavelengths.

Notably, on the SIMBA map, the dust peaks MMS 23 and the Coronet containing star formation appear to have faint emission 


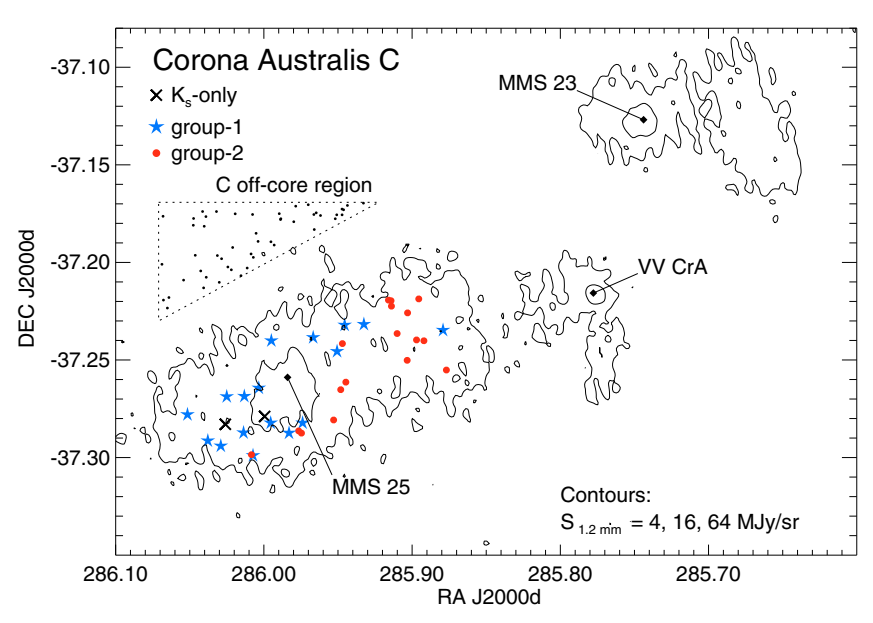

Fig. 8. Sky position of the possible members towards MMS 25 in field C. The dotted triangle encircles the stars in the $\mathrm{C}$ off-core region used for comparions. The off-field contains 12 group-1 and 55 group- 2 stars.

Table 4. Comparison of NW and C on- and off-core fields. The doublerows list the number of stars and the stellar surface density per $\operatorname{arcmin}^{2}$ with Poisson errors.

\begin{tabular}{ccccc}
\hline \hline & NW core & NW off & C core & C off \\
${\text { area/ } \operatorname{arcmin}^{2}}^{2}$ & 14.4 & 11.0 & 20.9 & 13.0 \\
\hline$K_{\mathrm{s}}<13$ & 12 & 13 & 19 & 17 \\
& $0.83 \pm 0.24$ & $1.18 \pm 0.33$ & $0.91 \pm 0.21$ & $1.31 \pm 0.32$ \\
$13<K_{\mathrm{s}}<15$ & 19 & 22 & 13 & 30 \\
& $1.32 \pm 0.30$ & $2.00 \pm 0.43$ & $0.62 \pm 0.17$ & $2.31 \pm 0.42$ \\
$K_{\mathrm{s}}$-only & 5 & \multicolumn{3}{c}{$0.10 \pm 0.07$} \\
& $0.35 \pm 0.16$ & & \\
\hline
\end{tabular}

tails extending more to the west than to the east (Figs. 4 and 8). The same holds for the quiet $1.2 \mathrm{~mm}$ source MMS 25 in cloud C. Also the $\mathrm{C}^{18} \mathrm{O}$ maps show these tails (Harju et al. 1993). We address possible implications in the final conclusions (Sect. 4).

To summarise, applying the projected mm-excess technique to our data yields: the Coronet contains about 53 stars $(\sim 20$ known +33 new $)$ in an area of $40 \operatorname{arcmin}^{2}$ having $S_{1.2 \mathrm{~m}}>25 \mathrm{MJy} / \mathrm{sr}$, yielding a stellar surface density of $1.3 / \mathrm{arcmin}^{2}$. In contrast, we find no evidence of candidate members in the NW and C cores. From the values in Table 4 we estimate that each of these cores contains at most 3 stars more massive than M4, corresponding to a stellar surface density of less than $0.3 / \operatorname{arcmin}^{2}$. This leads us to conclude that compared with the Coronet the NW and C core regions appear to be devoid of associated stars detectable on our near-infrared images.

\subsection{Search for K-band excess sources}

To identify young cloud members, which are detected in all three filters $J H K_{\mathrm{s}}$, a well-known strategy is to look for $K$-band excess sources by means of the colour-colour diagram $J-H$ versus $H-K_{\mathrm{s}}$. An excess of $K$-band emission is then interpreted as an additional contribution from hot $(T \sim 1500 \mathrm{~K})$ circumstellar dust, hence an indicator of pre-main-sequence objects that are likely associated with the parent molecular cloud. This strategy, however, requires relatively high accuracy in the photometry, the knowledge of the NIR reddening law, and no dilution of a $K$-band excess by scattering. So far only a few $K$-band excess sources have been found in the R Cr A cloud, virtually all of them bright stars (Wilking et al. 1997) saturated on our maps.
Unfortunately, our new probable members with projected mmexcess do not have $J H$ photometry. Here we have examined the sources fainter than $K_{\mathrm{s}}=11 \mathrm{mag}$. The slope of the $A_{\mathrm{V}}$ direction in the colour-colour diagrams has been derived from several sub-sets.

To summarise, based on our $J H K_{\mathrm{s}}$ data we find about a dozen sources having a small $K$-band excess compared to the reddened main sequence (Fig. 9, top). However, all these sources are faint (Fig. 9, bottom) and the statistical significance for $K$ band excess among them is marginal $(<3 \sigma)$. The photometric uncertainty as derived from the source extraction tools does not account for additional systematic errors that may be caused by unfavourable observing conditions. Therefore, we hesitate to claim the indicated $K$-band excess as real and call these sources $K$-band excess candidates. We just note that one source (RA/Dec B1950 = 18:58:53.3/-37:03:28) listed as a $K$-band excess source by Wilking et al. (1997) now turns out as a double source with $1^{\prime \prime} 6$ separation at PA $=175^{\circ}$ without evidence of any $K_{\mathrm{s}}$-band excess.

Our $K$-band excess candidates are located outside of the northwestern dust ridge (3-5 sources), south of the Coronet (3-4 sources), and southeast of VV CrA (4 sources). If they were associated with the R CrA cloud, then they would be very low-mass stars (Fig. 9, bottom). Notably, M-dwarfs with accretion and outflow signatures have been found 3.5 and 5!3 south and southwest of the Coronet by means of slitless optical spectroscopy and detailed follow-up studies (Fernández \& Comerón 2001, 2005; Barrado y Navascués et al. 2004). These very lowmass stars, lying outside the region of bright $1.2 \mathrm{~mm}$ emission, do not show any signs of $K$-band excess, either.

Obviously our NIR sources do not exhibit circumstellar discs with prominent hot dust emission showing up already in the $K$-band. Sources with cooler circumstellar discs may be found by combining our data with longer wavelength Spitzer data.

\subsection{Stars with nebulae and Herbig-Haro objects}

Apart from planetary nebulae, nebulous and extended objects are not expected among field stars. Hence such objects are probably associated with the star-forming molecular cloud.

The most conspicuous extended object is a beautiful X-shaped nebula, hereafter referred to as the "Isabelle-Nebula". With an extent of about $20^{\prime \prime}$ it is visible in $H$ and $K_{\mathrm{s}}$ but not in $J$. Figure 10 (top) shows the $K_{\mathrm{s}}$-band image. The sky-projected axis of the bipolar nebula is about perpendicular to the long axis of the entire R CrA cloud. While this could be a chance coincidence, a relation with the cloud's overall angular momentum also appears as an attractive explanation. The north-eastern part of the nebula appears somewhat brighter than the south-western part and has a prominent elongated emission along its northern edge. This bright spot has already been detected and denoted as IRAS 32c by Wilking et al. (1992). The position listed in Table 2 refers to the centre of the X-shape. It is coincident with the prominent millimetre point source MMS 23 (Chini et al. 2003) alias IRAS18595-3712 located about 16.3 southeast of R Cr A. Figure 10 (bottom) shows the $1-1200 \mu \mathrm{m}$ spectral energy distribution (SED). The SED is complemented in the mid-infrared by ISO 6.7 and $14.3 \mu \mathrm{m}$ photometry (Olofsson et al. 1999) and photometry at 3.6-24 $\mu \mathrm{m}$ was derived from the Spitzer archive using the basic calibrated data $(\mathrm{BCD})$ products version $\mathrm{S} 16$. In addition, far-infrared IRAS data are shown, supplemented by data points from SCUBA at 450 and $850 \mu \mathrm{m}$ (Nutter et al. 2005) and SIMBA at $1200 \mu \mathrm{m}$ (Chini et al. 2003). At first glance, the integrated $1-1200 \mu \mathrm{m}$ luminosity of about $2.1 L_{\odot}$ suggests a 

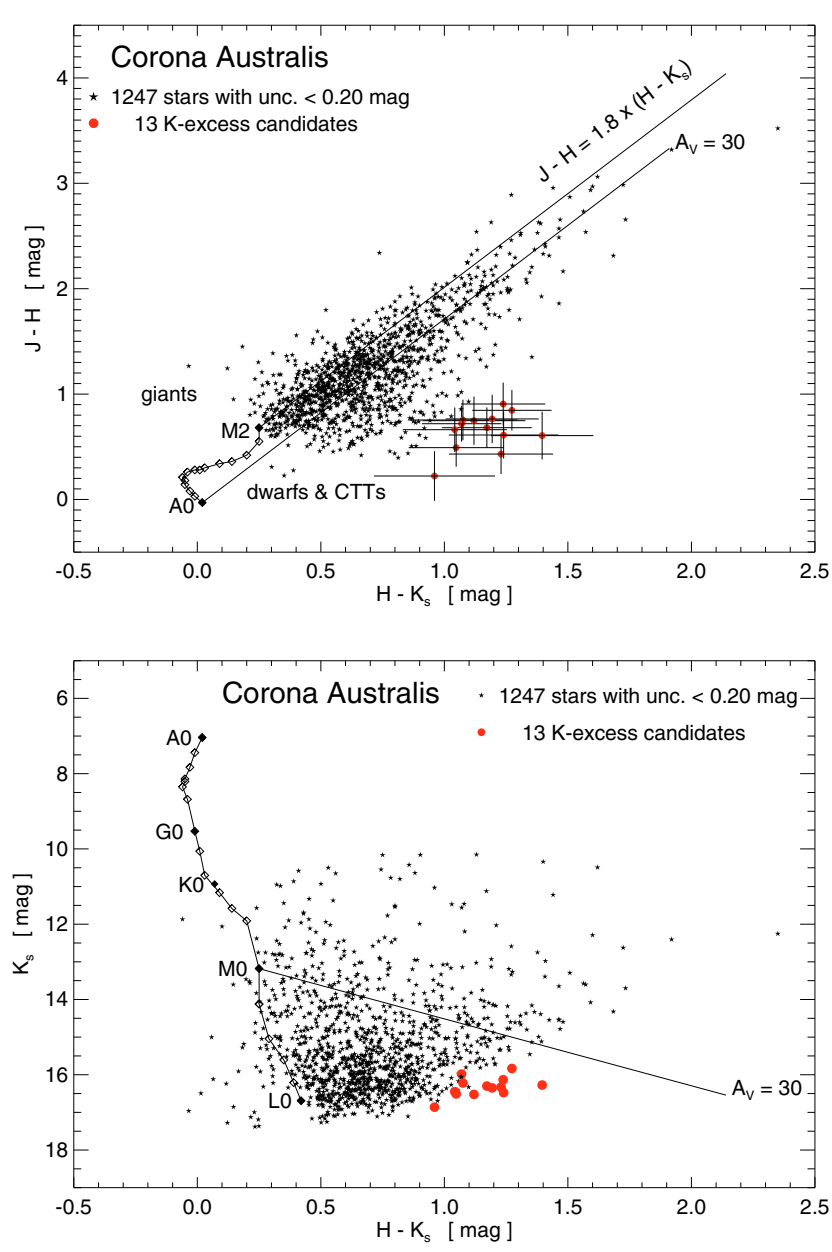

Fig. 9. Colour-colour and colour-magnitude diagrams showing the $K$-band excess candidates. The statistical significance for a $K$-band excess is low because of the large $1 \sigma$ error bars in the colour-colour diagram. In the colour-magnitude diagram the $K$-band excess candidates lie at the faint end of our sources.

solar mass PMS object. Obviously, the star exciting the Isabelle Nebula is deeply hidden and not directly visible at wavelengths shorter than $10 \mu \mathrm{m}$. The SED longwards of $10 \mu \mathrm{m}$ may mimic a pure class I object. However, the regular X-shape of the Isabelle Nebula suggests obscuration by a disc-like dust distribution seen roughly edge-on. The near-mid-infrared $(2-8 \mu \mathrm{m})$ hook of the SED cannot be interpreted as direct stellar radiation; it is instead produced by scattered light from the nebula. Furthermore, if the $H$ - and $K_{\mathrm{s}}$-band fluxes are dominated by continuum and not by line emission, the SED of the nebula appears to be strongly reddened by foreground dust. When disentangling such a nonspherical geometry, the actual luminosity of the PMS object increases significantly, because 1) the dust covering factor of the disc as seen from the central star is smaller $(\sim 50 \%)$ than that of the sphere, 2) the scattered near-mid-IR light comes from a narrow bi-cone with opening half-angle $30^{\circ}$ illuminated by the star; and 3 ) the near-mid-IR nebula is strongly reddened. Altogether, the actual luminosity increases potentially by a factor $4-10$, resulting in about $8-20 L_{\odot}$, consistent with a transition Class III object of spectral type F seen edge-on. While our coarse luminosity estimate is in line with results from SED fitting tools $\left(5-10 L_{\odot}\right.$ at intermediate inclinations, Robitaille et al. 2007), future NIR spectra and radiative transfer models constrained to the
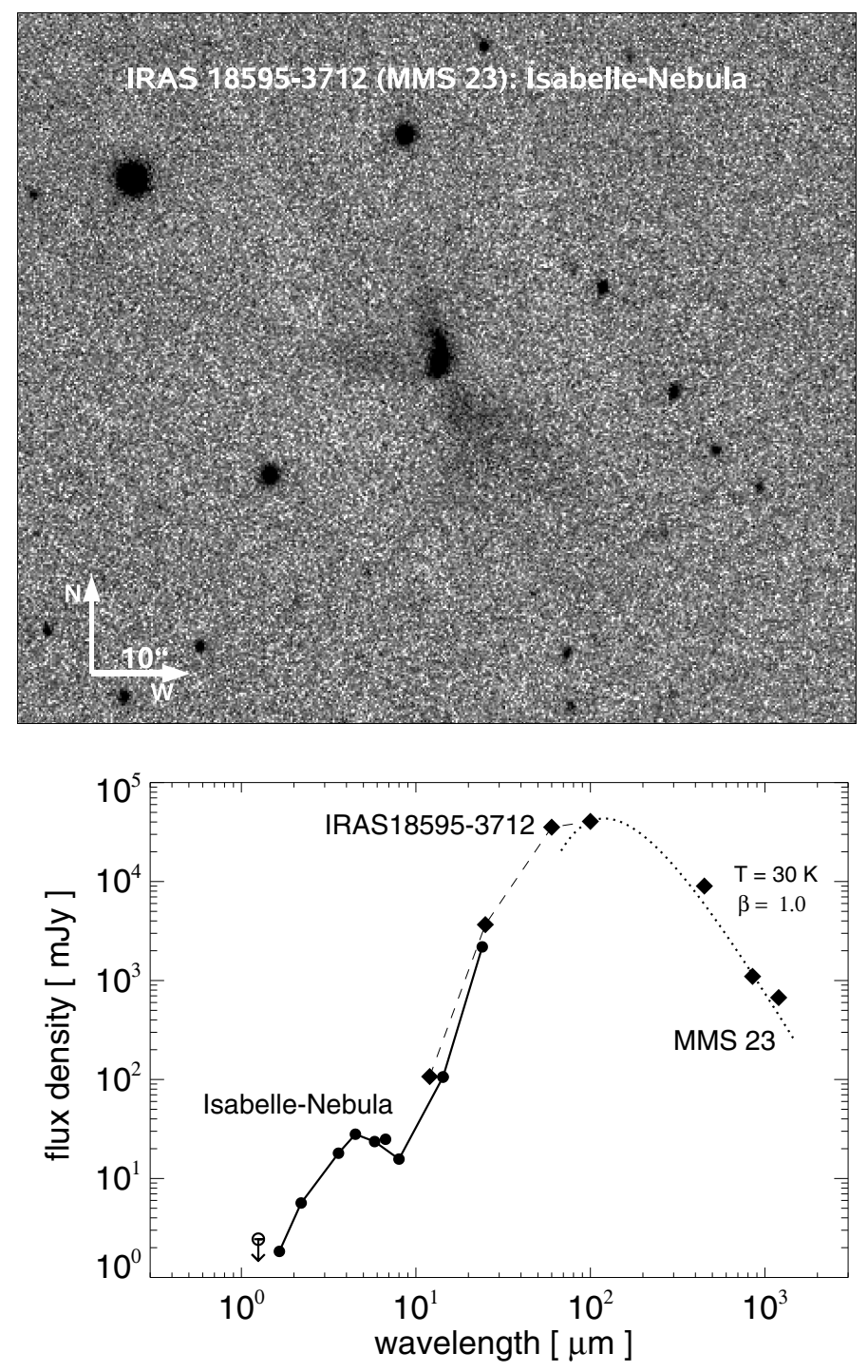

Fig. 10. $K_{\mathrm{s}}$-band image and SED of the X-shaped Isabelle Nebula. The lines and symbols in the SED plot mean: filled circles connected by the solid line mark our NIR data together with Spitzer and ISOCAM MIR data; the filled diamonds connected with a long-dashed line mark IRAS data; SCUBA $450+850 \mu \mathrm{m}$ and SIMBA $1.2 \mathrm{~mm}$ photometry is plotted with filled diamonds; the dotted line represents a modified blackbody with emissivity index $\beta=1$ and temperature $T=30 \mathrm{~K}$.

edge-on case will be needed to make any strong statements about the nature of this PMS star.

Another five objects fainter than $K_{\mathrm{s}}=10 \mathrm{mag}$ show small $\left(3-5^{\prime \prime}\right)$ elongated halos around the central star. They could not be resolved further in our images. At the distance of R CrA their NIR brightness indicates subsolar luminosities. We confirm the two sources listed as nebulous by Wilking et al. (1997). The sky position of the nebulae is shown in Fig. 11. In addition to the faint diffuse nebulae, we note that the bright Herbig Ae/Be-star TY CrA exhibits a remarkably sharp sickle-like double shell indicating multiple outbursts.

When looking at Herbig-Haro objects, HH 99 has a clear bow-shock arrow cap (Fig. 12). Drawing a mid-axis through the regular shape of $\mathrm{HH} 99$ suggests that either R Cr A or IRS 9 is its energy source. Because R Cr A can be excluded as discussed by Wilking et al. (1997), we suggest that IRS 9 is the most likely driving source for HH99. IRS 6 (located about 50" northwest 


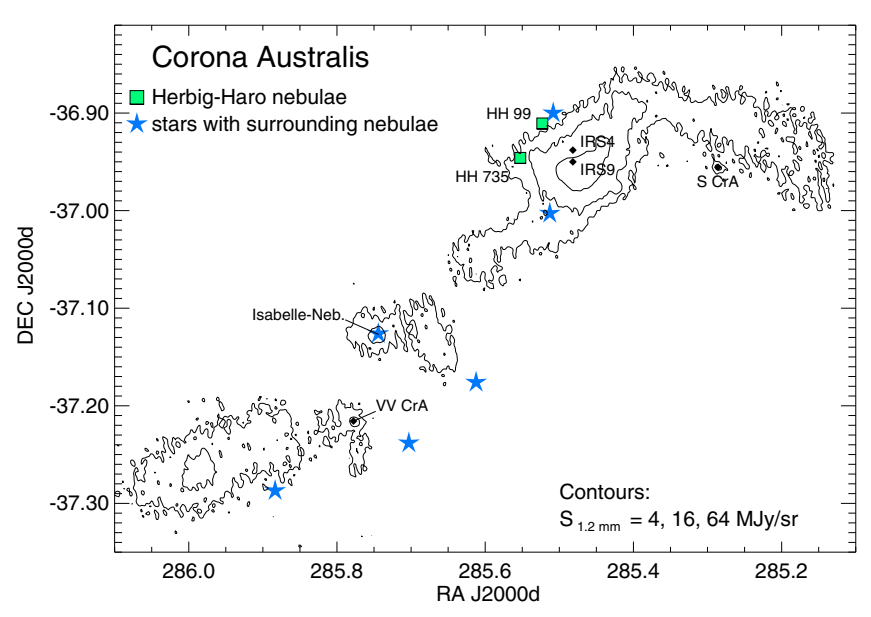

Fig. 11. Sky positions of the resolved nebulae.

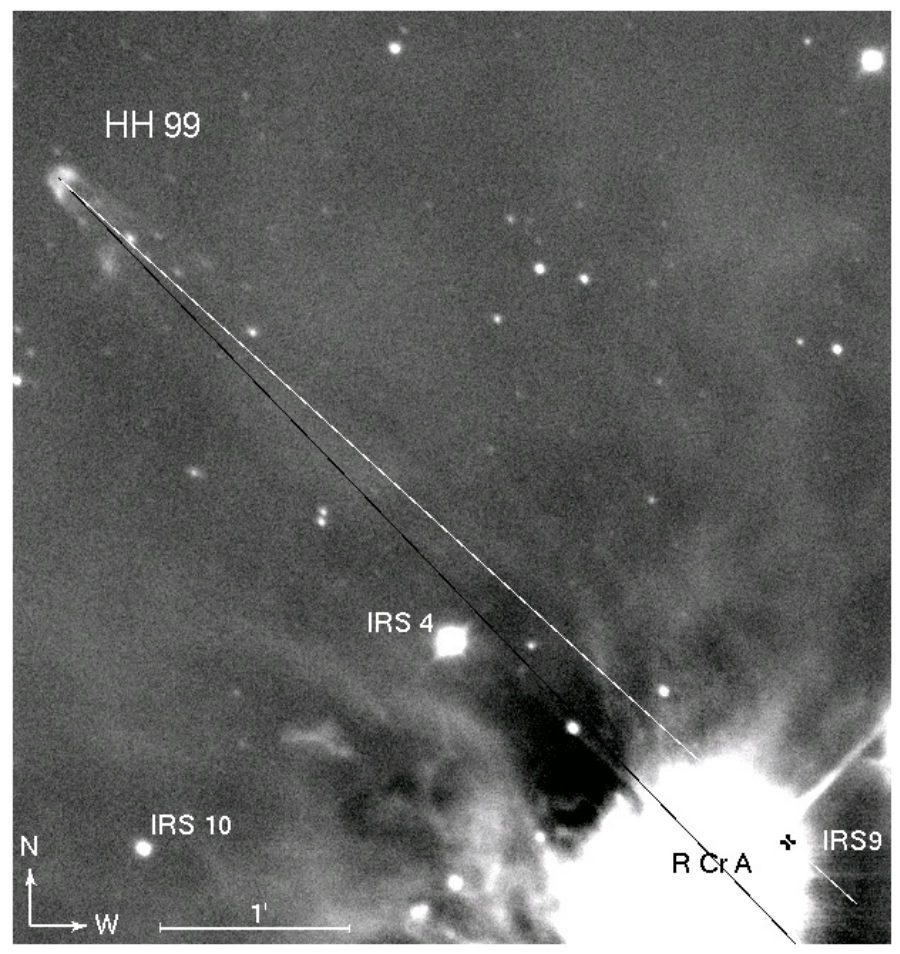

Fig. 12. $K_{\mathrm{s}}$-band image of $\mathrm{HH} 99$ with its bow-shock arrow cap. The black and white lines point to its driving star candidates R CrA and IRS 9 (marked with a "+” sign, right of R CrA), respectively. Because the bright stars are saturated in our frames, the position of the sources has been taken from Wilking et al. (1997) and Taylor \& Storey (1984). The positional uncertainty is about the size of the "+" sign.

of $\mathrm{R} \mathrm{CrA}$ ) as the driver of HH99, as proposed by Wang et al. (2004), may be questioned on the basis of alignment.

In general, the $K_{\mathrm{s}}$-band flux of the Herbig-Haro objects is probably dominated by the shocked molecular hydrogen line $\mathrm{H}_{2} 1-0 \mathrm{~S}(1)$ at $2.121 \mu \mathrm{m}$ and not by hot dust emission (e.g. Wilking et al. 1997, McCoey et al. 2004). Similarly, the $J$ and $H$-band covers the FeII lines at 1.257 and $1.644 \mu \mathrm{m}$, respectively. Our deep $K_{\mathrm{s}}$-band image of field A shows several more Herbig-Haro objects. For example, HH 732 A-C, located about $5^{\prime}$ north of R Cr A and HH 735, located about 25" north of the SIMBA point source MMS 19. Based on alignment with the [SII] $\lambda 6763 \AA$ contours elongated at PA $220^{\circ}$, Wang et al. (2004) suggested that HH 735 is driven by IRS 7 located 3.5 at PA = $260^{\circ}$. However, the optical data may suffer from patchy extinction. Drawing a mid-axis to the quite symmetric sickle shape seen in all three near-infrared bands points towards IRS 4 as a potential driving source. Most detected Herbig-Haro objects are located outside the area of bright $1.2 \mathrm{~mm}$ emission $\left(S_{1.2}>\right.$ $25 \mathrm{MJy} / \mathrm{sr}$ ), hence in a medium of low-moderate dust density. HH-861, HH-861, and HH863 detected with some caveats by Wang et al. (2004) around VV CrA are outside the FOV of our NIR maps in the southeastern part.

To summarise, while Herbig/Haro objects appear to be mainly concentrated around the coronet cluster, some faint stars with (compact) nebulae are found also towards the southeastern part of the elongated Corona Australis cloud (Fig. 11).

\section{Conclusions}

We performed a deep near-infrared survey of the entire R CrA molecular cloud and analysed the data with respect to the $1.2 \mathrm{~mm}$ dust continuum map.

1) Applying the projected mm-excess technique we find 33 new sources deeply embedded in the front layer towards the Coronet. Nine sources could be detected at $H$ and $K_{\mathrm{s}}, 24$ only have $K_{\mathrm{s}}$-band data. In contrast to the Coronet, the NW dust ridge and the $\mathrm{C}$ core region appear to be devoid of associated stars detectable in our near-infrared images. This strengthens the evidence for the Coronet being the region where most of the Class I-III stars are found. Obviously, core C contains at most very young protostars, which are too faint and embedded to be visible at near-infrared wavelengths. Stringent estimates about the contamination from background objects may be derived from Spitzer MIR data.

2) Only marginal evidence of $K$-excess sources can be found from our data $\left(K_{\mathrm{s}}>10 \mathrm{mag}\right)$. If these $K$-excess candidates are associated with the R CrA cloud, then they are very lowmass stars. In general, it seems that our sources do not exhibit circumstellar discs with prominent hot dust emission showing up already in the $K$-band.

3) The four new nebulae found towards the southeastern part corroborate the picture that star formation has also started in these regions.

4) The dust and CO peaks A Coronet and B (MMS 23) with ongoing star formation exhibit relatively sharp eastern borders and shallow western tails; the same holds for cloud C. If the star formation in the diverse $\mathrm{R} \mathrm{CrA}$ subcondensations were directly triggered by the wind from the Upper CentaurusLupus OB association, such a morphology is not expected at first glance. Instead in a scenario where an external wind hits an originally symmetric gas clump, one would expect that the front side, where the wind hits the clump, appears compressed and houses the first and stronger star formation. Because this is not observed, the picture is supported that the expanding Upper Centaurus-Lupus HI shell just may have led to fragmentation of the R CrA cloud into subcondensations, which further collapse to form stars mainly under the control of their own gravitation.

Our results are in line with the predictions by Harju et al. (1993) that the R CrA cloud has fragmented into subcondensations that are at different star-forming stages.

Acknowledgements. We thank Katrin Kämpgen for preparing the observations, Nicole van der Bliek and Marcus Albrecht for performing the observations, 
and Markus Nielbock for intriguing discussions, in particular on the HerbigHaro objects and nebulae. We also thank Bruce Wilking and Jochen Eislöffel for sending us their electronic $J H K$ and $R I$-band data files for comparison. The analysis greatly benefitted from the Aladin tool. This publication makes use of data products from the Two Micron All Sky Survey, which is a joint project of the University of Massachusetts and the Infrared Processing and Analysis Center/California Institute of Technology, funded by the National Aeronautics and Space Administration and the National Science Foundation. We thank the anonymous referee for the constructive, detailed report. This work was supported by the Nordrhein-Westfälische Akademie der Wissenschaften.

\section{References}

Anderson, I., Harju J., Knee L., \& Haikala L. 1997, A\&A, 321, 575 Barrado y Navascués, D., Mohanty, S., \& Jayawardhana, R. 2004, ApJ, 604, 284 Bertin, E., \& Arnouts, S. 1996, A\&AS, 117, 393

Brown, A. 1987, ApJ, 322, L31

Chini, R., Kämpgen, K., Reipurth, B., et al. 2003, A\&A, 409, 235

Dahn, C., Harris, H., Vrba, F., et al. 2001, AJ, 124, 1170

Ducati, J., Bevilacqua, C., Rembold, S., \& Ribeiro, D. 2001, ApJ, 558, 309

Fernández, M., \& Comerón, F. 2001, A\&A, 380, 264
Fernández, M., \& Comerón, F. 2005, A\&A, 440, 1119

Forbrich, J., \& Preibisch, T. 2007, A\&A, 475, 959

Harju, J., Haikala, L., Mattila, K., et al. 1993, A\&A, 278, 569

Hartigan, P., \& Graham, J. A.1987, AJ, 93, 913

Knude, J., \& Hog, E. 1998, A\&A, 338, 897

Krügel, E. 2003 The physics of interstellar dust, IoP Series in Astron., \& Astrophys. Bristol

Landolt-Börnstein 1982, Stars \& star clusters (Springer)

López Martí, B., Eislöffel, J., \& Mundt R. 2005, A\&A, 444, 175

Mc Coey, C., Giannini, T., Flower, D., et al. 2004, MNRAS, 353, 813

Marraco, H. G., \& Rydgren, A. E. 1981, AJ, 86, 62

Nutter D., Ward-Thompson D., \& André P. 2005, MNRAS, 357, 975

Olofsson, G., Huldtgren, M., Kaas, A. A., et al. 1999, A\&A, 350, 883

Robitaille, T. P., Whitney, B. A., Indebetouw, R., \& Wood, K. 2007, ApJS, 169, 328

Rossano, G. 1978, AJ, 83, 234

Schmidt-Kaler, T. 1982, in Landolt-Börnstein: Stars \& star clusters (Spinger)

Seward, F. D.1999, in Astrophysical Quantities, (AIP Press), ed. A. N. Cox, 183

Taylor, K. N. R., \& Storey, J. W. V.1984, MNRAS, 209, 5P

Wang, H., Mundt, R., Henning, T., \& Apai D. 2004, ApJ, 617, 1191

Wilking, B., Greene, T., Lada, C., et al. 1992, ApJ, 397, 520

Wilking, B., McCaughrean, M., Burton, M., et al. 1997, AJ, 114, 2029 